\title{
Measuring and Guaranteeing Quality of Barrier-Coverage in Wireless Sensor Networks
}

\author{
Ai Chen \\ Department of Computer \\ Science and Engineering \\ The Ohio State University \\ Columbus, $\mathrm{OH} 43210$ \\ chen.1109@osu.edu
}

\author{
Ten H. Lai \\ Department of Computer \\ Science and Engineering \\ The Ohio State University \\ Columbus, $\mathrm{OH} 43210$ \\ lai@cse.ohio-state.edu
}

\author{
Dong Xuan \\ Department of Computer \\ Science and Engineering \\ The Ohio State University \\ Columbus, $\mathrm{OH} 43210$ \\ xuan@cse.ohio-state.edu
}

\begin{abstract}
Sensors may fail due to various reasons such as heat, malicious activity, environmental hazards, extended use, and lack of power. As more and more sensors fail, certain desired properties such as barrier coverage will diminish and eventually fall below a desired level. In such a case, the network will have to be repaired. It is therefore desirable to have mechanisms to monitor network properties. In this paper, we are interested in measuring the quality of barrier coverage. In the literature, researchers only consider whether or not a sensor network provides barrier coverage. This is equivalent to measuring its quality as either 0 or 1 . We believe quality of barrier coverage is not binary and propose a metric for measuring it. If the measured quality is short of a desired value, we further identify all local regions that need to be repaired. The identified regions are minimum in the sense that if one of them is not repaired then the resulting network will still be short of quality. We also discuss how to actually repair a region.
\end{abstract}

\section{Categories and Subject Descriptors}

C.2.1 [Computer-Communication Networks]: Network Architecture and Design-network topology;

C.2.2 [Computer-Communication Networks]: Network Protocols

\section{General Terms}

Algorithms, Theory

\section{Keywords}

Wireless sensor networks, barrier coverage, quality, measuring, repairing.

\section{INTRODUCTION}

Recently there has been tremendous interest in monitoring borders with sensor networks. In a recent experiment,

Permission to make digital or hard copies of all or part of this work for personal or classroom use is granted without fee provided that copies are not made or distributed for profit or commercial advantage and that copies bear this notice and the full citation on the first page. To copy otherwise, to republish, to post on servers or to redistribute to lists, requires prior specific permission and/or a fee.

MobiHoc'08, May 26-30, 2008, Hong Kong SAR, China.

Copyright 2008 ACM 978-1-60558-073-9/08/05 ...\$5.00.
American Border Patrol placed sensors along the Mexican border near Sierra Vista. The sensors, called Seismic Intrusion Detectors, were buried and could pick up vibrations from foot steps within a certain range (NBC news, July 22, 2003).

Sensors deployed for this sort of application are said to provide barrier coverage or border coverage [5, 8, 13]. Specifically, in [8], a sensor network deployed over a belt region (such as international borders) is said to provide $k$-barrier coverage if every path that crosses the width of the belt is covered by at least $k$ distinct sensors. That is, $k$-barrier coverage guarantees to detect any intruder crossing the belt (border) along any path by at least $k$ sensors. The notion of $k$-barrier coverage is generalized in [5] to $L$-local $k$-barrier coverage that guarantees the detection of all crossing paths whose trajectory is confined to a slice (of length $L$ ) of the belt region. It is a generalization in the sense that when $L$ equals the length of the entire belt, $L$-local barrier coverage becomes the regular barrier coverage.

Sensors may fail due to various reasons such as heat, malicious activity, environmental hazards, extended use, and most of all, lack of power. A common approach to tolerating such failures is to deploy many redundant sensors $[6$, $7,12]$. However, no matter how many redundant sensors are deployed, they can only tolerate failures up to a certain degree. As more and more sensors fail, certain desired properties such as barrier coverage will diminish and eventually fall below a desired level. In such a case, the network will have to be repaired to restore the desired property. It is thus desirable to have mechanisms to monitor network properties. If sensor failures cause the network to fall below a desired level of "quality", we want to detect it in time. If it is deemed that the network indeed needs repair, we want to know which parts of the network need repair. It will not be cost effective to redeploy the entire network if, for example, adding a few sensors to some "weak" parts will be sufficient. We are interested in barrier coverage, and the above considerations have motivated us to consider the following problem.

Problem Statement: Given a sensor deployment over a belt region and a required quality $Q^{*}$, we wish to measure the quality of the deployment, $Q_{k}$, for $k$-barrier coverage. If the measured quality is short of the desired level (i.e. $\left.Q_{k}<Q^{*}\right)$, then the sensor network needs to be repaired. In this case, we wish to identify all weak sections that need to be repaired. The identified sections should have two properties: (1) if all of them are repaired, the quality of $k$-barrier 
coverage will be restored to $Q^{*} ;(2)$ if any of them is not repaired, the quality will still be short of $Q^{*}$ (i.e., no false weak sections are identified). To the best of our knowledge, this problem has not been addressed in the literature. This work is the first to discuss quality of barrier coverage.

Our first question is how to measure the quality of a network for barrier coverage. Consider the two sensor deployments in Figure 1. Neither of them provides 2-barrier coverage, as there exist crossing paths that are not covered by at least two sensors. However, even though neither network provides $100 \%$ 2-barrier coverage, it is apparent that network $B$ provides much better barrier coverage than network $A$. We are interested in a metric that will assign a value to a sensor network to indicate its quality of barrier coverage.

(A)

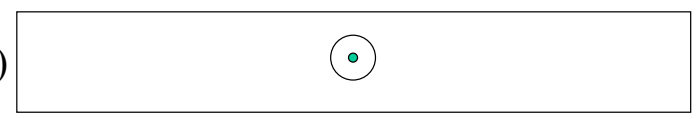

(B)

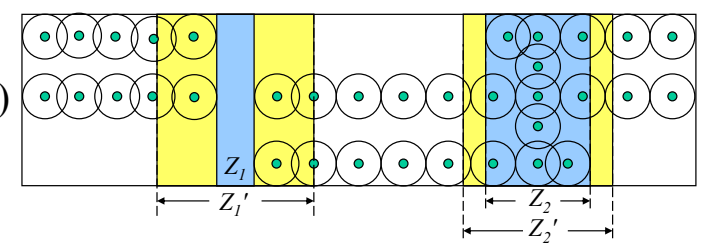

Figure 1: How to measure quality of barrier coverage?

Summary of Results: In this paper, we propose to measure the quality of $k$-barrier coverage for a belt region as the maximum value of $L$ such that the belt is $L$-local $k$ barrier covered ${ }^{1}$. Denote the quality so measured by $Q_{k}$. If $Q_{k}$ falls below a desired value $Q^{*}$, then we identify a set $R_{k}$ of all zones or sections of the belt that need to be repaired. Strictly speaking, it is not $Q_{k}$ alone but $Q_{k}$ and $R_{k}$ together that indicate the network's quality of $k$-barrier coverage. Applying our mechanism to the two deployments in Figure 1 will yield $Q_{2}(A)=Q_{2}(B)<0$. $R_{2}(A)$ will consist of a single section equal to the entire belt. $R_{2}(B)$ will comprise $Z_{1}^{\prime}$ and $Z_{2}^{\prime}$, which contain the weak zones $Z_{1}$ and $Z_{2}$ respectively; the lengths of $Z_{1}^{\prime}$ and $Z_{2}^{\prime}$ will depend on the value of $Q^{*}$. Our contributions in this paper can be summarized as follows.

- We introduce the concept of quality of barrier coverage. In the literature, researchers only consider whether or not a sensor network provides barrier coverage. This is equivalent to measuring its quality as either 0 or 1 . We believe that there are many different levels of quality, not just 0 and 1 .

- We propose a metric for measuring the quality of $k$ barrier coverage, and establish theorems that can be used to precisely measure the quality using the proposed metric.

- If the measured quality is short of a desired value, we have theorems that enable us to identify all local zones

\footnotetext{
${ }^{1}$ Two other metrics we have considered are: (1) the number of sensors needed to make the belt $k$-barrier covered and (2) the probability that an intruder following a randomly picked path will be detected by at least $k$ sensors. The latter seems appealing, but it is mathematically difficult to compute such probability.
}

that need to be repaired. The identified zones are minimum in the sense that if one of them is not repaired then the resulting network will still be short of quality.

- We show that the proposed theory can be realized as a distributed algorithm that measures the quality of $k$-barrier coverage and identifies regions to repair.

- We also discuss possible approaches to actually repairing a zone.

Relationship to Previous Work: The concept of $L$ local barrier coverage is introduced in [5]. Using it as a metric to measure the quality of $k$-barrier coverage is our idea. Methods to compute $Q_{k}$ (the quality) and produce $R_{k}$ (the sections to repair) are our contributions. In some cases, the theorems in [5] can be used to provide a lower bound on $Q_{k}$ (but not $Q_{k}$ itself); in many other cases, they can not even provide a lower bound. We wish to point out that the purpose of [5] is not to measure $Q_{k}$; as such, if the theorems in [5] do not provide a good measure of $Q_{k}$, it is not their weakness. We will elaborate on this with theoretical analysis and simulation results in Section 7.

Organization: The rest of this paper is organized as follows. Section 2 mentions some related work, and Section 3 describes the network model. Sections 4 and 5 constitute the theoretical foundations of measuring quality of barrier coverage and repairing weak zones. Section 6 describes our distributed algorithm that guarantees quality of barrier coverage. Performance study appears in Section 7. Section 8 concludes the paper.

\section{RELATED WORK}

The concept of barrier coverage is introduced in [8]. An interesting algorithm to determine whether a network provides barrier coverage is provided there. A centralized, optimal sleep-wakeup algorithm for achieving global barrier coverage is proposed in [9]. The notion of local barrier coverage is proposed in [5]. This new notion of barrier coverage is practically more appealing than the original barrier coverage, and it enables the authors of [5] to develop localized sleep-wakeup algorithms that provides near-optimal performance, while ensuring global barrier coverage most of the time. The problem of deriving a reliable estimate for ensuring 1-barrier coverage in a random deployment is solved in [3].

Several papers have discussed the problem of adding sensors to an existing deployment to achieve various properties, mostly $k$-connectivity. In [4], an algorithm is proposed that computes the minimum number of additional sensors needed to make a sensor network $k$-connected. The quality of $k$-connectivity considered there is either 1 or 0 , i.e., "yes, it is $k$-connected" or "no, it is not." The metric of $k$ redundancy is introduced in [1] to measure the local quality of $k$-connectivity; additional nodes (robots) are added to regions with low redundancy. Our work shares with [1] a similar flavor of measuring local quality and identifying regions that need repairs.

\section{PRELIMINARY AND NETWORK MODEL}

This section describes the network model. Many definitions are adopted from [5] and [8]. 
We assume that a sensor network is deployed over a belt region. An example belt region is illustrated in Figure 2. To formally define a belt region, let $d(x, y)$ denote the Euclidean distance between two points $x$ and $y$; and for a point $x$ and a curve $l$, let $d(x, l)$ be the distance between $x$ and $l$, i.e., $d(x, l)=\min \{d(x, y): y \in l\}$. Two curves $l_{1}$ and $l_{2}$ are said to be parallel with separation $w$ if $d\left(x, l_{2}\right)=d\left(y, l_{1}\right)=w$ for all $x \in l_{1}$ and $y \in l_{2}$.

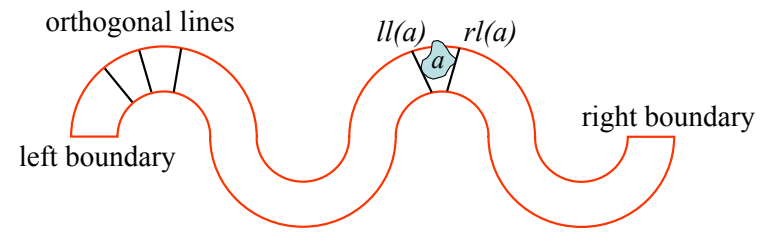

Figure 2: Example belt region, orthogonal lines, sensing region, leftmost/rightmost orthogonal lines.

Definition 3.1. [Belt region] A belt (region) has four boundaries. Two of them are parallel, and the other two are orthogonal to the two parallel ones. For ease of presentation, one of the orthogonal boundaries is referred to as the left boundary and the other the right boundary .

Definition 3.2. [Middle line] The middle line of a belt is the curve that is parallel to, at the middle between, the belt's two parallel boundaries. Its two ends are referred to as the left and the right endpoints.

DEFINITION 3.3. [Crossing paths and orthogonal lines] Intrusion movement is assumed to follow a "crossing path", where a path is said to be a crossing path if it crosses from one parallel boundary to the other. A crossing path is orthogonal if its length is equal to the belt's width. Orthogonal crossing paths are straight lines and, therefore, often referred to as orthogonal crossing lines or simply orthogonal lines.

The belt region can be viewed as the union of all orthogonal crossing lines. Each orthogonal crossing line intersects with the middle line at a unique point; different orthogonal crossing lines intersect the middle lines at different points. Thus, each orthogonal line $l$ can be assigned a "coordinate" as follows.

DeFinition 3.4. [Coordinate of an orthogonal line, $\left.V_{l}\right]$ For an orthogonal line $l$, let $V_{l}$ be the length of the middle line from the middle line's left endpoint to its intersection with $l$.

Assumption: We assume that the sensing region of a sensor $a$ is a continuous region surrounding $a$. It is not necessarily a disk.

DEFINITION 3.5. [Leftmost/rightmost orthogonal line of a sensing region] A sensing region's leftmost orthogonal line is the orthogonal line with smallest coordinate ( $V$ value) that has intersection with the sensing region. A sensing region's rightmost orthogonal line is similarly defined. The leftmost and rightmost orthogonal lines of a sensor node a's sensing region are denoted by $l l(a)$ and $r l(a)$, respectively.

Note that a sensing region's leftmost and rightmost orthogonal lines are unique.
Definition 3.6. [ $k$-Barrier coverage] A crossing path is $k$-covered if it intersects the sensing regions of at least $k$ distinct sensors. A sensor network $N$ provides $k$-barrier coverage over a belt region $B$ if all crossing paths through $B$ are $k$-covered by sensors in $N$.

Definition 3.7. [Zone] $A$ zone, $Z$, is a slice of the belt region. Two of its edges coincide with the belt's two parallel boundaries, and the other two edges are orthogonal crossing lines. The length of a zone $Z$, denoted by $S_{Z}$, is the length of the middle line in this zone.

Note that an orthogonal line is considered as a zone of length 0 .

Definition 3.8. [ $L$-Local $k$-barrier coverage] For $a$ nonnegative number $L$ and a positive integer $k$, a belt region is said to be L-local $k$-barrier covered if every zone of length $L$ in the region is $k$-barrier covered.

If a belt is $L$-local $k$-barrier covered, then for any $L^{\prime}$, where $0 \leq L^{\prime} \leq L$, it is also $L^{\prime}$-local $k$-barrier covered.

Definition 3.9. [Coverage graph, $\mathcal{G}(N)$ ] A coverage graph of a sensor network $N$ is defined as follows. Let $\mathcal{G}(N)=(V, E)$. The set $V$ consists of a vertex corresponding to each sensor. In addition, it has two virtual nodes, $s$ and $t$ corresponding to the left and right boundaries. An edge exists between two nodes if their sensing regions overlap in the deployment region. An edge exists between $u$ and $s$ (or $t$ ) if the sensing region of $u$ overlaps with the left boundary (or right boundary) of the region.

TheOREM 3.1. [8] A network $N$ provides $k$-barrier coverage iff there exist $k$ node-disjoint paths between the two virtual nodes $s$ and $t$ in $\mathcal{G}(N)$.

\section{MEASURING THE QUALITY OF BARRIER COVERAGE}

This section proposes a metric for measuring the quality of $k$-barrier coverage, shows how to measure it using that metric, and identifies all weak zones, if any, that need to be repaired.

DEFINITION 4.1. [Quality of $k$-barrier coverage, $Q_{k}$ ] The quality of a sensor deployment for $k$-barrier coverage, denoted by $Q_{k}$, is defined to be maximum $L$ such that the belt is $L$-local $k$-barrier covered; i.e. $Q_{k}=\max \{L$ : the belt is $L$-local $k$-barrier covered $\}$. If there is no such $L$ (i.e., if the belt is not even 0-local k-barrier covered), then define $Q_{k}=-1$.

In the rest of this section, it is assumed that we are given a positive integer $k$, a sensor deployment over a belt region $B$, and a desired quality $Q^{*}$. We will not repeat these in lemmas or theorems. Our first job is to compute $Q_{k}$.

$Q_{k}$ could be negative or nonnegative. We discuss these two cases separately. All definitions, lemmas and theorems apply to general belts and sensing models although rectangular belts and sensing disks are used for illustration in figures. 


\subsection{Is $Q_{k}=-1$ ?}

We first make a few definitions. Recall that each orthogonal line $l$ has a coordinate $V_{l}$ (Definition 3.4). For two orthogonal lines $l_{1}$ and $l_{2}$, we say that $l_{1}$ is on the left side of $l_{2}$ if $V_{l_{1}}<V_{l_{2}}$. Each sensor node $a$ is associated with two orthogonal lines $l l(a)$ and $r l(a)$ as defined in Definition 3.5. They will be simply referred to as the sensor's leftmost and rightmost orthogonal lines. We denote the left orthogonal boundary of a zone $Z$ by $l b(Z)$ and the right boundary by $r b(Z)$.

DEFINITION 4.2. [Zone from orthogonal line $l_{1}$ to $l_{2}$, $Z n\left(l_{1}, l_{2}\right)$ ] If two orthogonal lines $l_{1}$ and $l_{2}$ satisfy $V_{l_{1}} \leq V_{l_{2}}$, then the zone from $l_{1}$ to $l_{2}$ is denoted as $Z n\left(l_{1}, l_{2}\right)$; i.e. if $Z=Z n\left(l_{1}, l_{2}\right)$, then $l b(Z)=l_{1}$ and $r b(Z)=l_{2}$.

We first state an easy lemma that characterizes the case $Q_{k}=-1$.

LEMma 4.1. $Q_{k}=-1$ iff the belt contains an orthogonal crossing line that is not $k$-barrier covered.

Proof: The lemma follows directly from the definition of $Q_{k}$ and the fact that each orthogonal crossing line constitutes a zone of length 0 and vice versa.

We can not directly use Lemma 4.1 to check if $Q_{k}=-1$ since there are an infinite number of orthogonal crossing lines. We need a simpler necessary and sufficient condition for checking if $Q_{k}=-1$. The following definition is crucial for us to state such a condition.

Definition 4.3. [Sensing-boundary zone] Let $\mathcal{D}$ be the set of all sensors' left and right orthogonal lines, together with the belt's left and right orthogonal boundaries. Let $l_{0}, l_{1}, \ldots, l_{n}$ be all the lines in $\mathcal{D}$ as ordered from left to right, i.e., $V_{l_{0}}<V_{l_{1}}<\cdots<V_{l_{n}}$. (l $l_{0}$ and $l_{n}$ are the belt's left and right orthogonal boundaries, respectively.) We define $Z n\left(l_{i-1}, l_{i}\right)$ as a sensing-boundary zone $(1 \leq i \leq n)$.

As will be seen later, it is important to know whether a sensing-boundary zone is $k$-barrier covered. The following lemma provides a simple method.

Lemma 4.2. A sensing-boundary zone $Z n\left(l_{i-1}, l_{i}\right)$ is $k$ barrier covered iff there is a crossing path in $Z n\left(l_{i-1}, l_{i}\right)$ that has no intersection with $l_{i-1}$ and $l_{i}$ and is $k$ barrier covered.

Proof: The "only if" part is obvious. For the "if" part, suppose there is a $k$-barrier covered crossing path $p_{i}$ in $Z n\left(l_{i-1}, l_{i}\right)$ with no intersection with $l_{i-1}$ and $l_{i}$. Since $p_{i}$ is $k$-barrier covered, there are at least $k$ sensing regions intersecting with $p_{i}$. These regions must also intersect with both $l_{i-1}$ and $l_{i}$, since $Z n\left(l_{i-1}, l_{i}\right)$ is a sensing-boundary zone and $p_{i}$ has no intersection with $l_{i-1}$ and $l_{i}$. The entire zone $Z n\left(l_{i-1}, l_{i}\right)$ is therefore $k$-barrier covered.

The above lemma and the following theorem provide an easy way to check if $Q_{k} \neq-1$.

THEOREM 4.1. $Q_{k} \neq-1$ iff all sensing-boundary zones in the belt are $k$-barrier covered.

Proof: If all zones $Z n\left(l_{i-1}, l_{i}\right), 1 \leq i \leq n$, are $k$-barrier covered, then all orthogonal crossing lines are $k$-barrier covered and hence $Q_{k} \neq-1$. Conversely, if $Q_{k} \neq-1$, then all orthogonal crossing lines are $k$-barrier covered. In particular, for each $i, 1 \leq i \leq n$, the orthogonal crossing line at the middle of $Z n\left(l_{i-1}, l_{i}\right)$ (which evidently does not intersect $l_{i-1}$ and $\left.l_{i}\right)$ is $k$-barrier covered. By Lemma $4.2, Z n\left(l_{i-1}, l_{i}\right)$ is $k$-barrier covered.

\subsection{Computing $Q_{k}$ given $Q_{k} \neq-1$}

Now suppose that by applying Theorem 4.1, we know $Q_{k} \neq-1$. Our next job is to compute $Q_{k}$ given that $Q_{k} \neq-1$. We will introduce the concept of critical zones; establish the existence of such zones; and show that $Q_{k}$ equals the length of the shortest critical zone. A few definitions are in order.

DEFINITION 4.4. [Zone from node $a$ to node $b, Z n(a, b)$ ] If two nodes $a$ and $b$ are such that $V_{l l(a)} \leq V_{\text {rl(b) }}$, then we denote by $Z n(a, b)$ the zone from $l l(a)$ to $r l(b)$ and simply refer to it as the zone from $a$ to $b$. (Figure 3 shows an illustration.) If $V_{l l(a)}>V_{r l(b)}$, then $Z n(a, b)$ does not exist, in which case we write $Z n(a, b)=\emptyset$.

Note that for any two arbitrary nodes $a$ and $b$, either $Z n(a, b)$ or $Z n(b, a)$ exists. It is possible that both of them exist as illustrated in Figure 3. Also, $Z n(a, a)$ always exists for any node $a$. It is clear that $l b(Z n(a, b))=l l(a)$ and $r b(Z n(a, b))=r l(b)$ if $Z n(a, b) \neq \emptyset$.

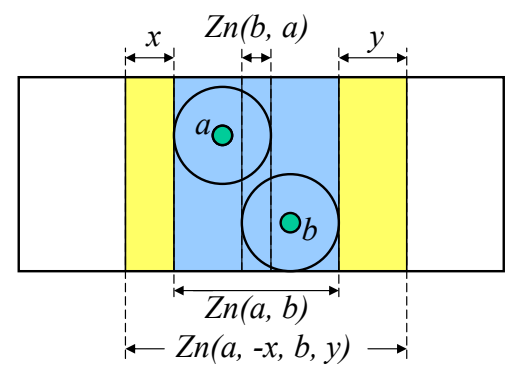

Figure 3: $Z n(a, b), Z n(b, a)$, and $Z n(a,-x, b, y)$

Definition 4.5. [Extended zone, $Z n(a,-x, b, y)$ ] If $Z n(a, b) \neq \emptyset$, for any $x \geq 0$ and $y \geq 0$, let $Z n(a,-x, b, y)$ denote the zone that is an extension of $Z n(a, b)$ by a length of $x$ in the left direction and a length of $y$ in the right direction. In other words, if $l_{1}$ and $l_{2}$ are the left and right boundaries of $Z n(a,-x, b, y)$, then $V_{l_{1}}=V_{l l(a)}-x$ and $V_{l_{2}}=V_{r l(b)}+y$ (Figure 3 shows an example.)

Note $Z n(a, 0, b, 0)=Z n(a, b)$. Let $B$ denote the entire belt. In the above definition, we implicitly assumed $Z n(a,-x, b, y) \subseteq B$. If $Z n(a,-x, b, y) \nsubseteq B$, then let $Z n(a,-x, b, y)=Z n(a,-x, b, y) \cap B$.

The concept of critical $k$-barrier covered zone will play an important role in our theory. Informally, a $k$-barrier covered zone is said to be critical if the zone will still be $k$-barrier covered when extended to the left or right by a small length; but will not be $k$-barrier covered if extended on both sides. Figure 4 shows a critical 1-barrier covered zone.

Definition 4.6. [Critical $k$-barrier covered zone] For two sensor nodes $a$ and $b$ such that $Z n(a, b) \neq \emptyset, Z n(a, b)$ is said to be $a$ critical $k$-barrier covered zone if the following conditions are all satisfied: (1) $Z n(a, b)$ is $k$-barrier covered; (2) there exists $a \delta>0$ such that $Z n(a,-\delta, b, 0)$ and 
$Z n(a, 0, b, \delta)$ are both $k$-barrier covered; (3) for any $\epsilon>0$, $Z n(a,-\epsilon, b, \epsilon)$ is not $k$-barrier covered. In addition, the entire belt region is regarded as a special critical $k$-barrier covered zone if it is $k$-barrier covered.

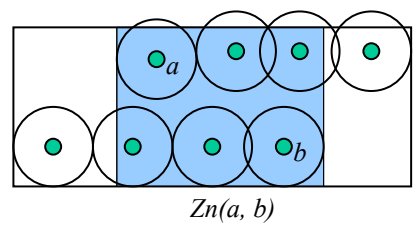

Figure 4: $Z n(a, b)$ is a critical 1-barrier covered zone, but is not a critical 2-barrier covered zone.

The following lemma follows directly from Definition 3.9 and Theorem 3.1. We state it as a lemma for ease of reference in subsequent proofs.

Lemma 4.3. Let $Z$ be a $k$-barrier covered zone, and let $Z^{\prime} \supseteq Z$ be another zone. If all sensing regions that intersect $l b(\bar{Z})$ or $r b(Z)$ also intersect $l b\left(Z^{\prime}\right)$ or $r b\left(Z^{\prime}\right)$, respectively, then $Z^{\prime}$ is also $k$-barrier covered.

We need the following definition in the next lemma.

Definition 4.7. For any zone $Z$, let $\operatorname{In}(Z)$ denote the "largest" subzone in $Z$ that is defined by two sensors and shares with $Z$ neither the left nor the right boundary. Thus, $\operatorname{In}(Z)=Z n(a, b) \subset Z$ for some sensors $a$ and $b$; between $l b(Z)$ and $l b(Z n(a, b))$ there is no any sensor's leftmost orthogonal line; and between $\operatorname{rb}(Z)$ and $\operatorname{rb}(Z n(a, b))$ there is no any sensor's rightmost orthogonal line. If In $(Z)$ does not exist, we write $\operatorname{In}(Z)=\emptyset$. (Figure 5 shows an illustration.)

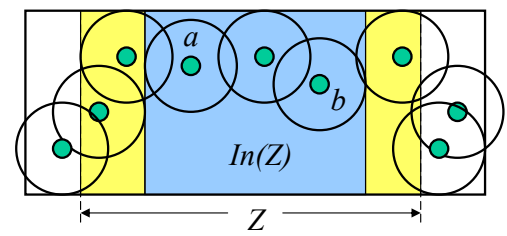

Figure 5: $\operatorname{In}(Z)=Z n(a, b)$

Weak zones must be contained in non- $k$-barrier covered zones. The next two lemmas explore the structures of non$k$-barrier covered zones and show that in every non- $k$-barrier covered zone there is a critical zone. This will establish the existence of critical $k$-barrier covered zones.

Lemma 4.4. If $Q_{k} \neq-1$, then for any non- $k$-barrier covered zone $Z, \operatorname{In}(Z) \neq \emptyset$.

Proof: It suffices to show that sensors $a$ and $b$ as in Definition 4.7 exist and $V_{l l(a)} \leq V_{r l(b)}$ so that $Z n(a, b)$ exists.

We first show the existence of $a$ and $b$. Since $Q_{k} \neq-1$, $r b(Z)$ is $k$-barrier covered (by Lemma 4.1 ). Thus, there are at least $k$ sensing regions overlapping with $r b(Z)$. At least one of these sensing regions, say $S_{x}$, does not overlapping with $l b(Z)$, as otherwise $Z$ would be $k$-barrier covered (by Lemma 4.3) and lead to a contradiction. Sensing region $S_{x}$ is on the right side of $l b(Z)$. Hence, the set $\left\{V_{l l(c)}: V_{l l(c)}>\right.$ $\left.V_{l b(Z)}\right\}$ is not empty and has a minimum. The sensor that corresponds to this minimum is node $a$. Similarly, node $b$ exists that satisfies $V_{r l(b)}=\max \left\{V_{r l(c)}: V_{r l(c)}<V_{r b(Z)}\right\}$.

Next we show $Z n(a, b) \neq \emptyset$. For contradiction, assume $Z n(a, b)=\emptyset$ (i.e. $\left.V_{r l(b)}<V_{l l(a)}\right)$. Let $l$ be an orthogonal crossing line in $Z$ that is between $r l(b)$ and $l l(a)$. Since $l$ is $k$-barrier covered, there are at least $k$ sensing regions overlapping with $l$. These regions must all overlap with $l b(Z)$; for if one of them, say $S_{x}$, does not overlap with $l b(Z)$, then $l l(x)$ will lie between $l b(Z)$ and $l l(a)$, i.e. $V_{l l(a)}>V_{l l(x)}>V_{l b(Z)}$, contradicting the fact that $V_{l l(a)}=\min \left\{V_{l l(c)}: V_{l l(c)}>\right.$ $\left.V_{l b(Z)}\right\}$. Similarly, the $k$ sensing regions (that overlap with $l$ ) must also overlap with $r b(Z)$. Since at least $k$ sensing regions overlap with both $l b(Z)$ and $r b(Z), Z$ is $k$-barrier covered, which is a contradiction. Therefore, $\operatorname{In}(Z)=Z n(a, b) \neq \emptyset$. $\square$

LEMmA 4.5. If $Q_{k} \neq-1$, then any non-k-barrier covered zone $Z$ contains a critical $k$-barrier covered subzone.

Proof: Since $Q_{k} \neq-1$, all orthogonal crossing lines are $k$-barrier covered by Lemma 4.1 .

Start with $Z$ and consider $\operatorname{In}(Z), \operatorname{In}(\operatorname{In}(Z))$, and so on. Repeatedly apply Lemma 4.4 and we will eventually reach a zone $Z^{\prime}$ such that $Z^{\prime}$ is not $k$-barrier covered but $\operatorname{In}\left(Z^{\prime}\right)$ is $k$-barrier covered. For simplicity, write $Z^{\prime}$ and $\operatorname{In}\left(Z^{\prime}\right)$ as $Z$ and $\operatorname{In}(Z)$, respectively, in the following.

Let $\operatorname{In}(Z)=Z n(a, b)$. If $Z n(a, b)$ is critical, the lemma is proved. So, assume $Z n(a, b)$ is not critical. There are two possible reasons for $Z n(a, b)$ to be not critical.

Case 1. $Z n(a,-\delta, b, \delta)$ is $k$-barrier covered for some $\delta>0$. Assume $Z n(a,-\delta, b, \delta) \subseteq Z$ (otherwise, choose a smaller $\delta$ to satisfy this assumption). Any sensing region overlapping with $l b\left(Z n\left(a,-\delta^{\prime}, b, \delta^{\prime}\right)\right)$ must also overlap with $l b(Z)$, due to the property of $Z n(a, b)=\operatorname{In}(Z)$. Similarly, any sensing region overlapping with $r b\left(Z n\left(a,-\delta^{\prime}, b, \delta^{\prime}\right)\right)$ must also overlap with $r b(Z)$. If $Z n(a,-\delta, b, \delta)$ is $k$-barrier covered, then by Lemma $4.3, Z$ is also $k$-barrier covered, which is a contradiction. So, this case is impossible.

Case 2. There exits a direction (left or right), such that for any $\delta>0$, when $Z n(a, b)$ is extended by the length of $\delta$ in that direction, the extended zone is not $k$-barrier covered. Without loss of generality, assume it is the right direction and so $Z n(a, 0, b, \delta)$ is not $k$-barrier covered for any $\delta>0$. Let $\delta>0$ be sufficiently small such that between the right boundary of $Z n(a, b)$ and that of $Z n(a, 0, b, \delta)$ there is no any sensor's leftmost or rightmost orthogonal line. (See Figure 6, where $Z_{1}=Z n(a, 0, b, \delta)$.) We construct a critical $k$-barrier covered zone as follows.

Let $l_{1}$ and $l_{r}$ denote the left and right boundaries of $Z n(a, 0, b, \delta)$, respectively. Consider all sensors' leftmost orthogonal lines between $l_{1}$ and $l_{r}$, including $l_{1}$ but not $l_{r}$. Let $l_{1}, \ldots, l_{n}$ be these lines as ordered from left to right (i.e., $\left.V\left(l_{1}\right)<V\left(l_{2}\right)<\cdots<V\left(l_{n}\right)\right)$. For $1 \leq i \leq n$, let $Z_{i}$ denote the zone from $l_{i}$ to $l_{r}$, i.e. $Z_{i}=Z n\left(l_{i}, l_{r}\right)$. Note that $Z n(a, 0, b, \delta)=Z_{1} \supseteq \cdots \supseteq Z_{n}$.

Claim: there is an index $i$ such that $Z_{i-1}$ is not $k$-barrier covered but $Z_{i}$ is. To prove this, it suffices to show that $Z_{1}$ is not $k$-barrier covered, but $Z_{n}$ is $k$-barrier covered. The former is obvious since $Z_{1}=Z n(a, 0, b, \delta)$. Now consider $Z_{n}$, whose left and right boundaries are $l_{n}$ and $l_{r}$. Since $l_{r}$ is $k$-barrier covered, there are at least $k$ sensing regions overlapping with $l_{r}$. These regions must also overlap with $l_{n}$ since there are no sensors' leftmost orthogonal lines between 
$l_{n}$ and $l_{r}$. Therefore, $Z_{n}$ is $k$-barrier covered. This proves the claim.

Let $i$ be the index mentioned in the above claim. Let $f_{i}$ and $f_{i-1}$ be sensor nodes such that $l l\left(f_{i}\right)=l_{i}$ and $l l\left(f_{i-1}\right)=$ $l_{i-1}$. (See Figure 6). We will show that $Z n\left(f_{i}, b\right)$ is a critical $k$-barrier covered zone.

Note that $Z n\left(f_{i}, b\right) \neq \emptyset$ and $Z_{i}=Z n\left(f_{i}, 0, b, \delta\right)$. So, as a subzone of $Z_{i}, Z n\left(f_{i}, b\right)$ is $k$-barrier covered; and there is an $\delta_{r}>0$ (e.g., $\delta_{r}=\delta$ ) such that $Z n\left(f_{i}, 0, b, \delta_{r}\right)$ is $k$-barrier covered. Similarly, as a subzone of $Z n(a, b), Z n\left(f_{i-1}, b\right)$ is $k$-barrier covered, and so there is a $\delta_{l}>0$ such that $Z n\left(f_{i},-\delta_{l}, b, 0\right)$ is $k$-barrier covered. It remains to show that for any $\epsilon>0, Z n\left(f_{i},-\epsilon, b, \epsilon\right)$ is not $k$-barrier covered.

For any $\epsilon>0$, choose $0<\epsilon^{\prime} \leq \epsilon$, such that $Z n\left(f_{i}, b\right) \subset$ $Z n\left(f_{i},-\epsilon^{\prime}, b, \epsilon^{\prime}\right) \subseteq Z_{i-1}$. (Recall that $Z_{i-1}$ is not $k$-barrier covered.) Let $l_{1}^{\epsilon}$ and $l_{2}^{\epsilon}$ be the left and right boundaries of $Z n\left(f_{i},-\epsilon^{\prime}, b, \epsilon^{\prime}\right)$, respectively. Both $l_{1}^{\epsilon}$ and $l_{2}^{\epsilon}$ are each $k$ barrier covered. Any sensing region overlapping with $l_{1}^{\epsilon}$ also overlaps with $l_{i-1}$, and any sensing region overlapping with $l_{2}^{\epsilon}$ also overlaps with $l_{r}$. By Lemma 4.3 , if $Z n\left(f_{i},-\delta^{\prime}, b, \delta^{\prime}\right)$ is $k$-barrier covered, then $Z_{i-1}$ would also be $k$-barrier covered, which is a contradiction. Therefore, $Z n\left(f_{i},-\epsilon^{\prime}, b, \epsilon^{\prime}\right)$ is not $k$-barrier covered. Since $Z n\left(f_{i},-\epsilon, b, \epsilon\right) \supseteq Z n\left(f_{i},-\epsilon^{\prime}, b, \epsilon^{\prime}\right)$, $Z n\left(f_{i},-\epsilon, b, \epsilon\right)$ is also not $k$-barrier covered.

We have shown $Z n\left(f_{i}, b\right)$ to be a critical $k$-barrier covered zone, and clearly $Z n\left(f_{i}, b\right) \subseteq Z n(a, b) \subset Z$. The lemma is proved.

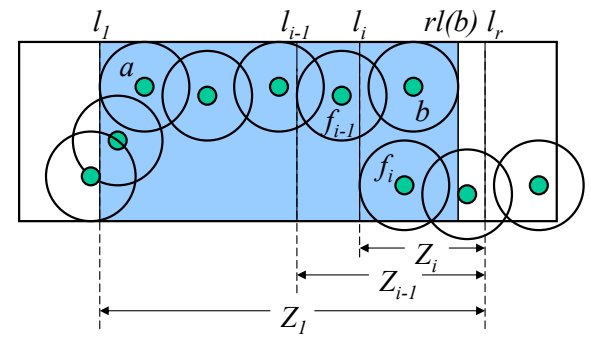

Figure 6: Visualizing the proofs of Lemma 4.5

As a result of Lemma 4.5, if $Q_{k} \neq-1$, then the belt contains at least one critical $k$-barrier covered zone. For if the whole belt is $k$-barrier covered, the belt itself is a critical $k$-barrier covered zone. If the whole belt is not $k$ barrier covered, it contains a critical $k$-barrier covered zone as indicated by the lemma. Thus, the following definition is meaningful.

Definition 4.8. If $Q_{k} \neq-1$, let $M_{k}$ denote the minimum length of critical $k$-barrier covered zones. That is, $M_{k}=\min \left\{S_{Z}: Z\right.$ is a critical k-barrier covered zone $\}$.

We are now ready to state the main result of this subsection: how to calculate $Q_{k}$ if $Q_{k} \neq-1$.

Theorem 4.2. If $Q_{k} \neq-1$, then $Q_{k}=M_{k}$.

Proof: It is obvious that $Q_{k} \leq M_{k}$. To show $Q_{k}=M_{k}$, assume otherwise that $Q_{k}<M_{k}$. Let $L$ be any value such that $Q_{k}<L<M_{k}$. By definition of $Q_{k}$, there exists a non$k$-barrier covered zone of length $L$. By Lemma 4.5, such a zone contains a critical $k$-barrier covered zone of length less than $L$ (and hence less than $M_{k}$ ). This contradicts the "minimum" property of $M_{k}$. Therefore, it must be the case $Q_{k}=M_{k}$.

Theorem 4.1 enables us to check if $Q_{k}=-1$. If $Q_{k} \neq-1$, Theorem 4.2 indicates that $Q_{k}$ equals the minimum length of critical $k$-barrier covered zones. A crucial question is then how to efficiently check whether zone $Z n(a, b)$ is a critical $k$ barrier covered zone. If $Z n(a, b)$ is the whole belt, just check whether it is $k$-barrier covered. If $Z n(a, b)$ is not the whole belt but it shares with the belt one orthogonal boundary, then $Z n(a, b)$ can not be a critical $k$-barrier covered zone since it can not simultaneously satisfy conditions 2 and 3 in Definition 4.6. For other cases, we have the following lemma. Due to space limit, we omit its proof. (The notation $\operatorname{In}(\cdot)$ used in the lemma is as defined in Definition 4.7.)

Lemma 4.6. Assume $Z n(a, b)$ shares no orthogonal boundary with the belt. $Z n(a, b)$ is a critical $k$-barrier covered zone iff there exist sensors $a^{\prime}$ and $b^{\prime}$ such that $Z n(a, b)=$ $\operatorname{In}\left(Z n\left(a^{\prime}, b^{\prime}\right)\right), Z n\left(a^{\prime}, b\right)$ and $Z n\left(a, b^{\prime}\right)$ are both $k$-barrier covered, and $Z n\left(a^{\prime}, b^{\prime}\right)$ is not $k$-barrier covered.

\subsection{Identifying all weak zones}

We use $Q_{k}$ to indicate the overall quality of $k$-barrier coverage in a belt region. Let $Q^{*}$ be the required quality. If $Q_{k} \geq Q^{*}$, the belt is in a good condition. If $Q_{k}<Q^{*}$, it is desired to identify all "weak" zones that do not provide adequate $k$-barrier coverage. Theorems 4.1 and 4.2 immediately suggest the following as weak zones:

- all non- $k$-barrier covered sensing-boundary zones;

- all critical $k$-barrier covered zones of length less than $Q^{*}$.

These zones can be easily identified by applying Lemma 4.2 and Lemma 4.6. They are each a weak zone because the existence of any of them will make $Q_{k}<Q^{*}$. Conversely, they comprise all weak zones in the sense that if all of them are "fixed" then $Q_{k}$ will become at least as high as $Q^{*}$. We will address the issue of "fixing weak zones" in the next section.

\section{REPAIRING WEAK ZONES}

Let $B$ be a belt region deployed with sensors for $k$-barrier coverage. Let $Q^{*}$ be the required quality. Suppose we have detected that $Q_{k}<Q^{*}$ and have identified all weak zones. Now we wish to repair these weak zones so that $Q_{k} \geq Q^{*}$. What does it mean to repair a weak zone? We clarify this notion in the following.

DEFINITION 5.1. [Repairing a zone] By repairing a zone $Z$, we mean adding new sensors to (and/or fixing existing failed sensors in) a zone $Z^{\prime} \supseteq Z$ such that certain objectives are achieved.

The significance of this definition is that to repair a zone $Z$, it may not be sufficient to just "repair" $Z$; we may have to actually "repair" a larger zone $Z^{\prime}$. Naturally, it is desired that $Z^{\prime}$ be as close to $Z$ as possible.

As mentioned in the preceding section, there are two types of weak zones: critical $k$-barrier covered zones of length less than $Q^{*}$ and non- $k$-barrier covered sensing-boundary zones. We consider them separately. 


\subsection{Dealing with critical $k$-barrier covered zones}

First, consider the case that the belt contains a critical $k$-barrier covered zone $Z n(a, b)$ with $S_{Z n(a, b)}<Q^{*}$. Intuitively, it may appear sufficient to consider a zone $Z \supset$ $Z n(a, b)$ of length $Q^{*}$ and make it $k$-barrier covered. That is not necessarily true as the following lemma indicates.

Lemma 5.1. Suppose the belt contains a (unique) critical $k$-barrier covered zone, $Z n(a, b)$, of length less than $Q^{*}$; and suppose now we add sensors to a zone $Z \supseteq Z n(a, b)$ such that $Z$ becomes $k$-barrier covered. If $S_{Z}<2 Q^{*}-S_{Z n(a, b)}$, then it is not necessary that $Q_{k} \geq Q^{*}$ (with the added sensors).

Proof: $\quad$ Since $Z n(a, b) \subseteq Z$, we view $Z$ as an extension of $Z n(a, b)$ on both the left and the right sides. Since $S_{Z}<$ $2 Q^{*}-S_{Z n(a, b)}$, either the left extension or the right extension (or both) must be of length less than $Q^{*}-S_{Z n(a, b)}$. Without loss of generality, assume the left extension has length less than $Q^{*}-S_{Z n(a, b)}$. Denote by $Z^{\prime}$ the zone $Z n(a, b)$ plus its left extension. Then, $S_{Z^{\prime}}<Q^{*}$. Now, slightly extend $Z^{\prime}$ to $Z^{\prime \prime}$ on both the left and the right sides such that $S_{Z^{\prime}}<S_{Z^{\prime \prime}}<Q^{*}$. Note that $Z n(a, b) \subset Z^{\prime \prime} \nsubseteq Z$. As a zone extended from $Z n(a, b)$ (which is critical) on both sides, $Z^{\prime \prime}$ is not $k$-barrier covered (before new sensors are added). We will show that adding sensors to make $Z k$-barrier covered does not necessarily make $Z^{\prime \prime} k$-barrier covered. To this aim, it suffices to construct an instance.

In order to make $Z k$-barrier covered, we add sensors in $Z$ to create $k$ disjoint paths between virtual nodes $s$ and $t$ in the coverage graph of $Z$ (see Definition 3.9 and Theorem 3.1). Place these sensors in a way such that their sensing regions do not intersect any existing sensors' sensing regions as well as the left boundary of $Z^{\prime \prime}$. As such, these added sensors do not create any new path in the coverage graph of $Z^{\prime \prime}$ between its virtual nodes $s^{\prime \prime}$ and $t^{\prime \prime}$. Hence, $Z^{\prime \prime}$ is still not $k$-barrier covered. As $S_{Z^{\prime \prime}}<Q^{*}$, we have $Q_{k}<Q^{*}$.

The next question is how much to extend zone $Z n(a, b)$ such that $Q_{k} \geq Q^{*}$ if the extended zone is made $Q^{*}$-local $k$-barrier covered. The following lemma provides an answer.

Lemma 5.2. Assume $Q_{k} \neq-1$. Let $Z n\left(a_{i}, b_{i}\right), 1 \leq i \leq n$ be all critical $k$-barrier covered zones with length less than $Q^{*}$, and let $\delta_{i}=Q^{*}-S_{Z n\left(a_{i}, b_{i}\right)}$. If sensors are added to the belt such that each $Z n\left(a_{i},-\delta_{i}, b_{i}, \delta_{i}\right)$ becomes $Q^{*}$-local $k$ barrier covered, then $Q_{k} \geq Q^{*}$

Proof: Let $Z$ be any zone of length $Q^{*}$. We need to show that after sensors are added, $Z$ is $k$-barrier covered. Suppose $Z$ is not $k$-barrier covered before sensors are added; otherwise we are done. By Lemma 4.5, $Z$ contains a critical $k$-barrier covered zone $Z^{\prime}$. Since $S_{Z^{\prime}}<S_{Z}=Q^{*}, Z^{\prime}$ equals one of the critical zones $Z n\left(a_{i}, b_{i}\right)$. It is clear that $Z \subseteq Z n\left(a_{i},-\delta_{i}, b_{i}, \delta_{i}\right)$. The latter is $k$-barrier covered after sensors are added. Hence, $Z$ is $k$-barrier covered after sensors are added.

\subsection{Dealing with non- $k$-barrier covered sensing-boundary zones}

Now, suppose the belt contains a sensing-boundary zone $Z$ that is not $k$-barrier covered and hence represents a weak zone. Suppose we repair a zone $Z^{\prime} \supseteq Z$ such that $Z^{\prime}$ becomes $Q^{*}$-barrier covered. The following lemma indicates that we need $S_{Z^{\prime}} \geq 2 Q^{*}+S_{Z}$. Due to space limit, we omit its proof since it is similar to the proof of Lemma 5.1.

Lemma 5.3. Suppose the belt $B$ contains a non-k-barrier covered sensing-boundary zone $Z$; suppose $B-Z$ is $Q^{*}$-local $k$-barrier covered; and suppose sensors are added to $B$ such that a zone $Z^{\prime} \supseteq Z$ becomes $k$-barrier covered. If $S_{Z^{\prime}}<$ $S_{Z}+2 Q^{*}$, then $Q_{k}$ may still be less than $Q^{*}$.

The next question is how much to extend these non- $k$ barrier covered sensing-boundary zones such that $Q_{k} \geq Q^{*}$ if we make all the extended zones $Q^{*}$-local $k$-barrier covered. The next lemma provides an answer.

Lemma 5.4. Let $Z_{i}, 1 \leq i \leq m$, be all non- $k$-barrier covered sensing-boundary zones in the belt $B$ such that $B-$ $\left(\cup_{i=1}^{m} Z_{i}\right)$ is $Q^{*}$-local $k$-barrier covered. Let $Z_{i}^{\prime}$ be the extension of $Z_{i}$ by a length of $Q^{*}$ on each of the left and right sides. If sensors are added to make each $Z_{i}^{\prime} Q^{*}$-local $k$ barrier covered, then $Q_{k} \geq Q^{*}$.

Proof: Let $Z$ be a zone with $S_{Z}=Q^{*}$. If $Z \cap Z_{i} \neq \emptyset$ for some $i$, then $Z \subset Z_{i}^{\prime}$ and hence $Z$ is $k$-barrier covered after repairing. If $Z \cap Z_{i}=\emptyset$ for all $i, 1 \leq i \leq m$, then $Z$ is $k$-barrier covered even before repairing.

\subsection{Repairing all weak zones}

The following theorem is essentially Lemmas 5.2 and 5.4 put together. It describes exactly what extended weak zones to repair.

TheOREM 5.1. Assume the belt B contains non-k-barrier covered sensing-boundary zones $Z_{i}, 1 \leq i \leq m$, and critical $k$-barrier covered zones $Z n\left(a_{j}, b_{j}\right), 1 \leq j \leq n$, of length less than $Q^{*}$. Let $Z_{i}^{\prime}$ be the extension of $Z_{i}$ by a length of $Q^{*}$ on each of the left and right sides, and let $\delta_{j}=Q^{*}-$ $S_{Z n\left(a_{j}, b_{j}\right)}$. If sensors are added to make each $Z_{i}^{\prime}$ and each $Z n\left(a_{j},-\delta_{j}, b_{j}, \delta_{j}\right) Q^{*}$-local $k$-barrier covered, where $1 \leq i \leq$ $m$ and $1 \leq j \leq n$, then $Q_{k} \geq Q^{*}$.

Proof: Consider $B-\left(\cup_{i=1}^{m} Z_{i}\right)$, which is a collection, say $\mathcal{S}$, of sub-belts of $B$. Each sub-belt in $\mathcal{S}$ contains no non$k$-barrier covered sensing-boundary zone. That is, $Q_{k} \neq-1$ for each belt in $\mathcal{S}$. If we make each $Z n\left(a_{j},-\delta_{j}, b_{j}, \delta_{j}\right) Q^{*}{ }_{-}$ local $k$-barrier covered, then by Lemma 5.2 , each belt in $\mathcal{S}$ is $Q^{*}$-local $k$-barrier covered. By further making each $Z_{i}^{\prime} Q^{*}$-local $k$-barrier covered, according to Lemma 5.4 , the whole belt $B$ becomes $Q^{*}$-local $k$-barrier covered and hence $Q_{k} \geq Q^{*}$.

\subsection{How to actually repair a zone?}

For a weak zone $Z$, once we have determined the extended zone $Z^{\prime} \supseteq Z$ on which to actually perform the repair, the next question is how to repair $Z^{\prime}$; i.e., how to make $Z^{\prime}$ $Q^{*}$-local $k$-barrier covered. We outline three possible approaches.

1) Replacement: Suppose the initial sensor network had the required quality $Q^{*}$, which however has diminished because of sensor failures. Then it suffices to just replace all failed sensor nodes in $Z^{\prime}$ with new motes (or just replace sensors and/or batteries as appropriate). This will make $Z^{\prime}$ $Q^{*}$-local $k$-barrier covered.

2) Random deployment: This method, which adds new sensor nodes to $Z^{\prime}$ at random locations, may be appealing 
if the initial sensor network was deployed randomly. The question of how many sensors to add seems not much different from that of determining the initial sensor density for the initial deployment, provided that sensors fail following a Poisson process. In this case, the results in [3] and [8] for determining sensor density may be applicable.

3) Deterministic deployment: Using this approach, one determines specific locations to place new sensors. An interesting combinatorial problem can be formulated as follows: Given a sensor deployment over a zone $Z^{\prime}$, determine the locations to place additional sensors such that $Z^{\prime}$ becomes $Q^{*}$-local $k$-barrier covered and the number of sensors required is minimum. This is still an open problem. However, a simpler version of it, which looks for the minimum number of additional sensors needed to make $Z$ ' " $k$-barrier covered" (as opposed to the more relaxed " $Q *$-local $k$-barrier covered"), can be solved in polynomial time. This simple version is adequate for our application.

\section{A PROTOCOL GUARANTEEING QUALITY OF BARRIER COVERAGE}

Based on results of the last two sections, we now describe a distributed algorithm that, given a sensor deployment over a belt for $k$-barrier coverage and a required quality $Q^{*}$, returns a (possibly empty) set of weak zones. Once these zones are repaired, the belt will provide $k$-barrier coverage with quality $Q^{*}$. If the set of weak zones is empty, the required quality is already met.

Assumptions: One important input to the algorithm is the belt region. We assume that the gateway has information about the shape of the entire region, but each sensor only knows a small section of it (more precisely, the section of length $Q^{*}+r_{\max }$ to the right of the sensor with $r_{\max }$ denoting the maximum sensing range if sensing ranges are different in different directions). One simple way to represent a belt region is by describing its midline and the width $W$. With the midline, it is easy to calculate orthogonal lines. In most papers on coverage (e.g., [2] and [8]) sensing regions are assumed to be disks. Our algorithm does not require sensing disks; instead, we assume that the sensing region is continuous and each node $a$ can calculate its leftmost and rightmost orthogonal lines, $l l(a)$ and $r l(a)$. The calculation of $l l(a)$ and $r l(a)$ do not have to be very precise, but the approximated (calculated) $l l(a)$ and $r l(a)$ must be between the theoretical $l l(a)$ and $r l(a)$. We also assume that given any two sensor nodes it is possible to know if their sensing regions have intersection. This assumption is necessary for any coverage problem and can be realized if every node knows its sensing region and its relative location to other nodes. Relative location can be obtained by using a localization algorithm such as the one in [10]. We assume that each node has a unique ID as is common in newer platforms such as TelosB [11]. There are gateways (or base stations) at certain places of the belt region. We assume that the network's communication graph is such that every node is able to communicate with neighbors and with at least one gateway. How to ensure network connectivity has been studied in [1] and [4]. A sensor node is either an active node or a failed node.

Algorithm GUARANTEE: the algorithm comprises two parts, one for every sensor node and the other for gateways. Every active node $a$ executes the following.
1) Node $a$ checks if the sensing-boundary zone $Z n(l l(a), l)$ that starts from $l l(a)$ is $k$-barrier covered by applying Lemma 4.2. If it is not, then include this zone in a report $R$ as a non- $k$-barrier covered sensing-boundary zone. (Note: it is easy for $a$ to compute $l$ since $Z n(l l(a), l) \subseteq$ $Z n(l l(a), r l(a))$.

2) There is a unique sensing-boundary zone $Z n\left(r l(a), l^{\prime}\right)$ that starts from $r l(a)$. This zone's right boundary $l^{\prime}$ may or may not be known by $a$. Thus, $a$ checks whether $Z n\left(r l(a), l^{\prime}\right)$ is a non- $k$-barrier covered sensing-boundary zone as follows:

- If there is no node in zone $Z n\left(r l(a), l^{\prime \prime}\right)$, where $V_{l^{\prime \prime}}=$ $V_{r l(a)}+\max \left\{Q^{*}, r_{\max }\right\}$, then include $\operatorname{Zn}(r l(a), ?)$ in the report $R$ as a non- $k$-barrier covered sensing-boundary zone, where "?" is used to indicate that the right boundary of this zone is not known by the reporting sensor a.

- If, otherwise, $a$ is able to gather information about $l^{\prime}$ from nodes in $Z n\left(r l(a), l^{\prime \prime}\right)$, then $a$ checks whether $Z n\left(r l(a), l^{\prime}\right)$ is $k$-barrier covered. If not, then include $Z n\left(r l(a), l^{\prime}\right)$ in the report $R$ as a non- $k$-barrier covered sensing-boundary zone.

3) Node $a$ checks if there is a critical $k$-barrier covered zone $Z n(a, b)$ of length less than $Q^{*}$, and if so, includes it in the report. (This can be easily done by checking for all $b$ with $S_{Z n(a, b)}<Q^{*}$, whether $Z n(a, b)$ is a critical $k$-barrier covered zone, by applying Lemma 4.6 . There can be at most one critical $k$-barrier covered zone $Z n(a, \cdot)$ with $a$ as the first parameter.)

4) After the above measurements, node $a$ sends the report $R$ to a gateway.

Actions of the gateway upon receiving a report: (There are possibly multiple gateways, which are assumed to be capable of communicating with one another. For simplicity, we will describe the algorithm as if there is only one gateway.)

1) If the objective of running the algorithm is just to measure the quality, then whenever the gateway receives a node $a$ 's report, it updates the value of $Q_{k}$ as follows: If the report includes a non- $k$-barrier covered sensing-boundary zone, then let $Q_{k}=-1$; otherwise, if critical zone $Z n(a, b)$ exists, let $Q_{k}=\min \left\{Q_{k}, S_{Z n(a, b)}\right\}$.

2) If the objective is to identify all weak zones that need to be repaired, then after a gateway gets the report from an active node $a$, it updates the list $L$ of "need-to-be-repaired" zones as follows.

- If any of $Z n(l l(a), l), Z n\left(r l(a), l^{\prime}\right)$ and $Z n(a, b)$ exist, just add them to $L$.

- If $Z n(r l(a), ?)$ was reported, a little more effort is needed for the gateway to find the zone's right boundary. Among all nodes that have sent in their reports by the end of the algorithm, let node $b$ be the node such that $l l(b)$ is on the right side of and is closest to $r l(a)$; then add $Z n(r l(a), l l(b))$ to $L$ as a non- $k$-barrier covered sensing-boundary zone. If such a node $b$ does not exist, then add $Z n\left(r l(a), l^{\prime}\right)$ to $L$ as a non- $k$-barrier covered sensing-boundary zone, where $l^{\prime}$ is the belt region's right boundary.

- There are two special cases that need to be taken care of toward the end of the algorithm. First, the gateway needs to check whether the sensing-boundary zone starting from 
the belt's left boundary is $k$-barrier covered; if not, add it to $L$. (The gateway has the information necessary to do this from all active nodes' reports.) Another special case, which probably won't happen in practice, is when the gateway does not receive any single report. This means that there is not any active node in the entire belt, and we simply add the entire belt to $L$ as a special "need-to-be-repaired" zone.

- At the end of the algorithm, $L$ contains the zones that need to be repaired. Note that for zones $Z \in L$, it is the extended zones $Z^{\prime} \supset Z$ as described in Theorem 5.1 that need to be actually repaired (i.e., made $Q^{*}$-local $k$-barrier covered).

Complexity analysis: Let $n$ be the maximum number of nodes in any zone of length $Q^{*}+r_{\max }$. In step 1 of the algorithm for sensors, zone $Z n(l l(a), l)$ can be found in $O(n)$ time. Whether the zone is $k$-barrier covered can be determined in $O\left(k^{2} n\right)$ time [8]. Similarly, step 2 can be done in $O\left(k^{2} n\right)$ time. In step 3, whether a zone $Z n(a, b)$ is a critical $k$-barrier covered zone can be determined by applying Lemma 4.6 in $O\left(k^{2} n\right)$ time. Using binary search, node $a$ needs to check at most $\log n$ zones. Thus, this step requires $O\left(k^{2} n \log n\right)$ time. So, totally, the running time for each node is $O\left(k^{2} n \log n\right)$.

\section{PERFORMANCE STUDY}

As mentioned in the introduction, the purpose of [5] is not to measure $Q_{k}$. However, based on the theorems proved there, it is not hard to design an algorithm for estimating $Q_{k}$ and identifying weak zones. Let us call such an algorithm SEEM. (Note: there is no such an algorithm in [5].) In this section, we wish to compare the performance of our algorithm, called GUARANTEE, and that of algorithm SEEM. The purpose of this comparison is to provide evidences that simply applying the theorems in [5] is not adequate for estimating $Q_{k}$ and identifying weak zones; there is a need for a more efficient and more effective algorithm, and our algorithm was intended to serve this purpose.

We will provide theoretical analysis as well as simulation results for our comparison. We have implemented algorithms GUARANTEE and SEEM in MATLAB, using a belt region of dimension $5,000 m \times 200 m$. Sensors were deployed randomly with uniform distribution. The sensing range $(r)$ is $30 \mathrm{~m}$, and $k=1$. The required quality $Q^{*}=200 \mathrm{~m}$. For every simulation case, 100 random scenarios were simulated.

\subsection{Measuring quality}

The basis of algorithm SEEM is Theorems 4.1 and 4.4 in [5]. We will only discuss Theorem 4.1 since it performs better than the other, which is for the general belt.

Theorem 4.1 in [5]: Let $r$ be the sensing range. If $d>r$ and every node's $2 d$-zone is $k$-barrier covered, the belt is $L$-local $k$-barrier covered where $L=\max \{d+r, 2 d-2 r\}$.

A node $a$ 's $2 d$-zone is a zone $Z_{a}$ with $S_{Z_{a}}=2 d$ and $a$ being at the middle of $Z_{a}$. Suppose we use algorithm SEEM, which is based on this theorem, to measure quality. It will calculate the maximum value of $d$ such that every node's $2 d$ zone is $k$-barrier covered; and then it will attempt to report the value of $Q_{k}$ according to $d$. Two undesired things may happen.

- First, if $d \leq r$, then algorithm SEEM will fail to say anything about $Q_{k}$. For example, the rectangular belt in Figure 7 has a bad quality of 1-barrier coverage since it is even not $2 r$-local 1-barrier covered. The maximum value of $d$ for nodes $a$ and $b$ is $r$; but SEEM cannot recognize the poor quality of coverage. In simulation, We varied the number of nodes from 500 to 1000 to study how often GUARANTEE and SEEM would report the quality of barrier coverage. The results are shown in Figure 8. GUARANTEE always reported the quality, which is guaranteed by our theoretical analysis. However, SEEM failed to report anything especially when the coverage quality was bad. For example, when the number of nodes was 500, it never reported any estimate of the quality. When the number of nodes was 900 , it only reported 72 times out of 100 scenarios.

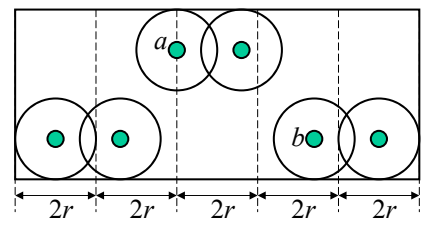

Figure 7: $Q_{1}<2 r$ but SEEM will fail to report it.

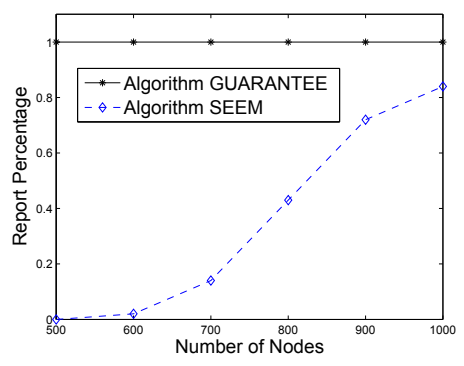

Figure 8: Quality report percentage.

- Second, if $d>r$, algorithm SEEM will report $Q_{k} \approx$ $\max \{2 d-2 r, d+r\}$, which actually is a lower bound on $Q_{k}$. For example, for the deployment in Figure 9, the actual value of $Q_{1}$ is $6 r$. The lower bound provided by SEEM will be $4 r$, since the maximum value of $d$ (for which every node's $2 d$ zone is $k$-barrier covered) is $3 r$. GUARANTEE will always report the precise value of $Q_{k}$.

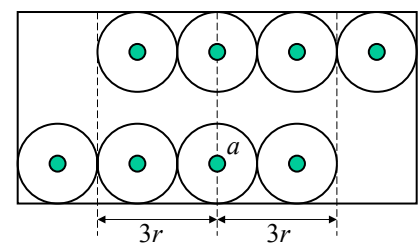

Figure 9: $Q_{1}=6 r$ but SEEM will report $Q_{1} \approx 4 r$.

\subsection{Identifying weak zones}

Now suppose we use algorithm SEEM to identify weak zones for repairs. Let the required quality be $Q^{*}$. According to Theorem 4.1 in [5], a node will report its $2 d$-zone as a weak zone if it is not $k$-barrier covered, where $d=\min \left\{Q^{*} / 2+\right.$ $\left.r, Q^{*}-r\right\}$. Three undesired things may happen.

- SEEM may ask for repairing even though it is unnecessary. For the rectangular belt in Figure 9, if the required 
quality is $6 r$, the network satisfies the requirement since the belt is $6 r$-local 1-barrier covered. But SEEM will report $4 r$ as the quality and unwarrantedly identify node $a$ 's $2 d$-zone as a weak zone. This drawback can be very costly especially if it requires human workers to do the repairs. GUARANTEE will never ask for unnecessary repairing.

- On the other hand, the weak zones identified by SEEM may not be complete, in the sense that even if all these zones are repaired, there is no guarantee that $Q^{*}$ will be reached. For example, the quality of the belt in Figure 1(A) is poor since it only contains one sensor node. However, SEEM will only report this node's $2 d$-zone as a weak zone. By making this $2 d$-zone $k$-barrier covered, the resulting quality $Q_{k}$ will still be -1 . GUARANTEE always identifies all weak zones needed to be repaired.

- Even if SEEM can repair the belt to reach the required quality (as it can in some cases), it will use more sensors than GUARANTEE. The reason is that the sizes of the weak zones identified by SEEM are larger than the sizes of the (extended) weak zones identified by GUARANTEE. We studied their differences in simulation. We considered the belt originally containing 1500 nodes and the quality met the required quality value $200 \mathrm{~m}$. Then, we varied the number of active nodes (as opposed to failed nodes) and used the replacement approach (as described in Section 5.4) to repair all weak zones. For every simulation case, 10 random scenarios were simulated. The results appear in Figure 10. GUARANTEE always required a less number of replaced nodes than SEEM. When the number of active nodes was 700, SEEM replaced 215.40 failed nodes on average, while GUARANTEE replaced only 174.50, $19 \%$ less.

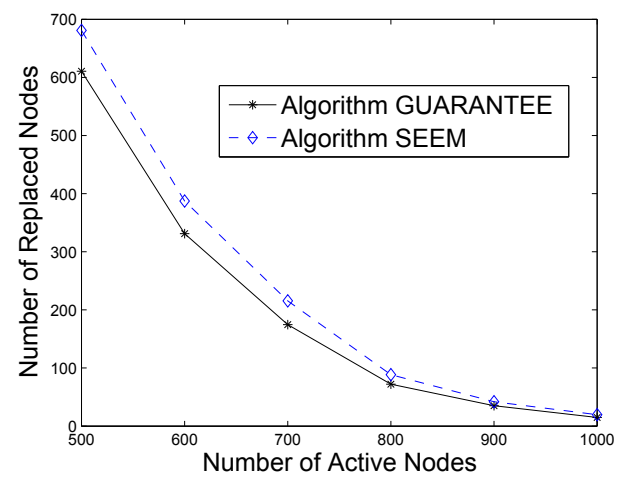

Figure 10: Number of replaced nodes to reach the required quality.

\section{CONCLUDING REMARKS}

We introduced the concept of "quality of barrier coverage" and proposed an effective way to measure it. If the measured quality falls below a desired level, we proposed a method to identify all weak zones that need to be repaired. Once these zones are repaired, the network's quality of barrier coverage will be restored to the desired level. The identified zones are optimal in the sense that if any of them is not repaired, the quality will still be short of the desired value.

Connectivity is another important property required of sensor networks. It is not clear what kind of connectivity is most suitable for barrier-coverage sensor networks. If $k$ - connectivity is desired as is assumed in many studies on connectivity of sensor networks, then recent results in [1] and [4] can be used to measure the quality of $k$-connectivity and identify regions to place additional nodes to either reestablish or enhance the quality of $k$-connectivity.

A straightforward way to use our algorithm and the algorithms in [1] and [4] to ensure the network's quality of $k_{1}$-barrier coverage and $k_{2}$-connectivity, is to run them separately. It will be an interesting problem to design a unified algorithm that monitors the network and suggests repairs to ensure $k_{1}$-barrier coverage and $k_{2}$-connectivity simultaneously.

\section{ACKNOWLEDGMENT}

This work was supported in part by the Army Research Office under grant No. AMSRD-ACC-R 50521-CI.

\section{REFERENCES}

[1] N. Atay and B. Bayazit. Mobile Wireless Sensor Network Connectivity Repair with K-Redundancy. Technical report, Washington University in St. Louis.

[2] X. Bai, S. Kumar, D. Xuan, Z. Yun, and T. H. Lai. Deploying Wireless Sensors to Achieve both Coverage and Connectivity. In Proc. of MobiHoc'06, 2006.

[3] P. Balister, B. Bollobas, A. Sarkar, and S. Kumar. Reliable Density Estimates for Achieving Coverage and Connectivity in Thin Strips of Finite Length. In Proc. of ACM Mobicom'07, Canada, September 2007.

[4] J. L. Bredin, E. D. Demaine, M. Hajiaghayi, and D. Rus. Deploying Sensor Networks with Guaranteed Capacity and Fault Tolerance. In Proc. of ACM MobiHoc'05, 2005.

[5] A. Chen, S. Kumar, and T. H. Lai. Designing Localized Algorithms for Barrier Coverage. In Proc. of ACM Mobicom'0\%, Canada, September 2007.

[6] Y. Gao, K. Wu, and F. Li. Analysis on the Redundancy of Wireless Sensor Networks. In Proc. of ACM WSNA'03, 2003.

[7] R. Iyengar, K. Kar, and S. Banerjee. Low-Coordination Topologies for Redundancy in Sensor Networks. In Proc. of MobiHoc'05, 2005.

[8] S. Kumar, T. H. Lai, and A. Arora. Barrier Coverage with Wireless Sensors. In Proc. of ACM MobiCom'05, 2005 .

[9] S. Kumar, T. H. Lai, M. E. Posner, and P. Sinha. Optimal Sleep Wakeup Algorithms for Barriers of Wireless Sensors. In Proc. of BROADNETS'07, 2007.

[10] M. Li and Y. Liu. Rendered Path: Range-Free Localization in Anisotropic Sensor Networks with Holes. In Proc. of ACM Mobicom'07, 2007.

[11] J. Polastre, R. Szewczyk, and D. Culler. Telos: Enabling Ultra-Low Power Wireless Research. In Proc. of IPSN'05, 2005.

[12] C. Schurgers, V. Tsiatsis, S. Ganeriwal, and M. Srivastava. Topology Management for Sensor Networks: Exploiting Latency and Density. In Proc. of MobiHoc'02, 2002.

[13] M. K. Watfa and S. Commuri. Power Conservation Approaches to the Border Coverage Problem in Wireless Sensor Networks. In Proc. of ICWN'06, Las Vegas, USA, June 2006. 